POS PROCEEDINGS

\title{
Observation of intense fluxes of charged particles in association with thundercloud in Tibet
}

M. Amenomori ${ }^{1}$, X. J. Bi ${ }^{2}$, D. Chen ${ }^{3}$, T. L. Chen ${ }^{4}$, W. Y. Chen ${ }^{2}$, S. W. Cui ${ }^{5}$,

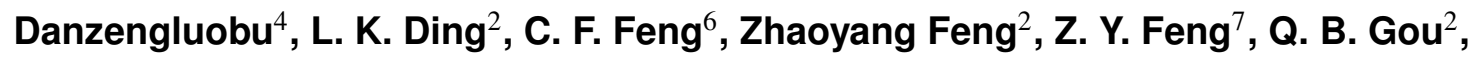
Y. Q. Guo ${ }^{2}$, H. H. He ${ }^{2}$, Z. T. He ${ }^{5}$, K. Hibino ${ }^{8}$, N. Hotta ${ }^{9}$, Haibing $\mathrm{Hu}^{4}$, H. B. $\mathrm{Hu}^{2}$, J. Huang ${ }^{2}$, H. Y. Jia ${ }^{7}$, L. Jiang ${ }^{2}$, F. Kajino ${ }^{10}$, K. Kasahara ${ }^{11}$, Y. Katayose ${ }^{12}$, C. Kato ${ }^{13}$, K. Kawata ${ }^{14}$, M. Kozai ${ }^{13}$, Labaciren ${ }^{4}$, G. M. Le ${ }^{15}$, A. F. Li ${ }^{16,6,2}$, H. J. Li ${ }^{4}$, W. J. Li ${ }^{2,7}$, C. Liu $^{2}$, J. S. Liu ${ }^{2}$, M. Y. Liu ${ }^{4}$, H. Lu ${ }^{2}$, X. R. Meng ${ }^{4}$, T. Miyazaki ${ }^{13}$, K. Mizutani ${ }^{11,17}$, K. Munakata ${ }^{13}$, T. Nakajima ${ }^{13}$, Y. Nakamura ${ }^{13}$, H. Nanjo ${ }^{1}$, M. Nishizawa ${ }^{20}$, T. Niwa ${ }^{13}$, M. Ohnishi ${ }^{14}$, I. Ohta ${ }^{19}$, S. Ozawa ${ }^{11}$, X. L. Qian ${ }^{6,2}$, X. B. Qu ${ }^{2}$, T. Saito ${ }^{21}$, T. Y. Saito ${ }^{22}$, M. Sakata ${ }^{10}$, T. K. Sako ${ }^{14}$, J. Shao ${ }^{2,6}$, M. Shibata ${ }^{12}$, A. Shiomi ${ }^{23}$, T. Shirai ${ }^{8}$, H. Sugimoto ${ }^{24}$, M. Takita ${ }^{14}$, Y. H. Tan ${ }^{2}$, N. Tateyama ${ }^{8}$, S. Torii ${ }^{11}$, H. Tsuchiya ${ }^{25}$, S. Udo ${ }^{8}$, H. Wang ${ }^{2}$, H. R. Wu ${ }^{2}$, L. Xue ${ }^{6}$, Y. Yamamoto ${ }^{10}$, K. Yamauchi ${ }^{12}$, Z. Yang ${ }^{2}$, S. Yasue ${ }^{26}$, A. F. Yuan ${ }^{4}$, T. Yuda ${ }^{14}$, L. M. Zhai ${ }^{2}$, H. M. Zhang ${ }^{2}$, J. L. Zhang ${ }^{2}$, X. Y. Zhang ${ }^{6}$, Y. Zhang ${ }^{2}$, Yi Zhang ${ }^{2}$, Ying Zhang ${ }^{2}$, Zhaxisangzhu ${ }^{4}$, X. X. Zhou ${ }^{7}$ 


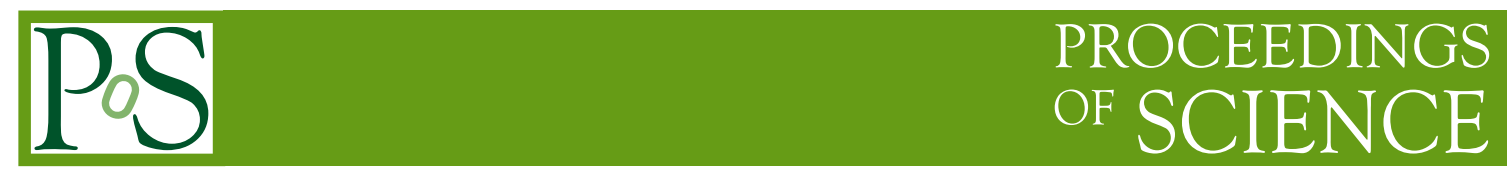

(The Tibet AS $\gamma$ Collaboration)

${ }^{1}$ Department of Physics, Hirosaki University, Hirosaki 036-8561, Japan

${ }^{2}$ Key Laboratory of Particle Astrophysics, Institute of High Energy Physics, Chinese Academy of Sciences, Beijing 100049, China

${ }^{3}$ National Astronomical Observatories, Chinese Academy of Sciences, Beijing 100012, China

${ }^{4}$ Department of Mathematics and Physics, Tibet University, Lhasa 850000, China

${ }^{5}$ Department of Physics, Hebei Normal University, Shijiazhuang 050016, China

${ }^{6}$ Department of Physics, Shandong University, Jinan 250100, China

${ }^{7}$ Institute of Modern Physics, SouthWest Jiaotong University, Chengdu 610031, China

${ }^{8}$ Faculty of Engineering, Kanagawa University, Yokohama 221-8686, Japan

${ }^{9}$ Faculty of Education, Utsunomiya University, Utsunomiya 321-8505, Japan

${ }^{10}$ Department of Physics, Konan University, Kobe 658-8501, Japan

${ }^{11}$ Research Institute for Science and Engineering, Waseda University, Tokyo 169-8555, Japan

${ }^{12}$ Faculty of Engineering, Yokohama National University, Yokohama 240-8501, Japan

${ }^{13}$ Department of Physics, Shinshu University, Matsumoto 390-8621, Japan

${ }^{14}$ Institute for Cosmic Ray Research, University of Tokyo, Kashiwa 277-8582, Japan

${ }^{15}$ National Center for Space Weather, China Meteorological Administration, Beijing 100081, China

${ }^{16}$ School of Information Science and Engineering, Shandong Agriculture University, Taian 271018, China

${ }^{17}$ Saitama University, Saitama 338-8570, Japan

${ }^{18}$ National Institute of Informatics, Tokyo 101-8430, Japan

${ }^{19}$ Sakushin Gakuin University, Utsunomiya 321-3295, Japan

${ }^{20}$ College of Science, China University of Petroleum, Qingdao, 266555, China

${ }^{21}$ Tokyo Metropolitan College of Industrial Technology, Tokyo 116-8523, Japan

${ }^{22}$ Max-Planck-Institut für Physik, München D-80805, Deutschland

${ }^{23}$ College of Industrial Technology, Nihon University, Narashino 275-8576, Japan

${ }^{24}$ Shonan Institute of Technology, Fujisawa 251-8511, Japan

${ }^{25}$ Japan Atomic Energy Agency, Tokai-mura 319-1195, Japan

${ }^{26}$ School of General Education, Shinshu University, Matsumoto 390-8621, Japan

E-mail: tibet.asqdgmail.com

To measure the correlation between thundercloud and atmospheric charged particles, we have installed some atmospheric electric field meter at a site on the Tibet Air shower Array $(4,300 \mathrm{~m}$ a.s.1.) since February 2010. In this paper, we report some results of coincident observation of data from the array and atmospheric electric field during thunderstorm. In addition, we present comparisons of a Monte Carlo simulation with the relativistic runaway electron avalanche in cosmic-ray air shower.

The 34th International Cosmic Ray Conference,

30 July- 6 August, 2015

The Hague, The Netherlands 